\title{
Dances to a redox tune
}

\author{
F. Garcia-Olmedo, M. Piñeiro and I. Diaz \\ Laboratorio de Bioquímica y Biología Molecular, E.T.S. Ingenieros Agrónomos-UPM, E-28040 Madrid, \\ Spain
}

Reduction and oxidation of proteins through changes in their disulfide/sulfhydryl groups (S-S/ $\mathrm{SH}$ ) have been increasingly implicated over the past 25 years in the redox regulation of plant metabolism in the light and in the dark. Recent findings indicate that this redox mechanism might be important in modulating all levels of gene expression in plants and suggest new avenues for future work.

The elucidation of the ferredoxin/thioredoxin system has been a major contribution to our knowledge of plant metabolism and is the best known example of redox regulation by the above mechanism [see 1]. In summary, thioredoxins undergo reversible $\mathrm{S}-\mathrm{S} / 2 \mathrm{SH}$ changes catalyzed by ferredoxin-thioredoxin reductase. Ferredoxin itself is reduced by the electron transport chain in thylakoid membranes in the light. The system works through the activation or inactivation of target enzymes by a net transfer of reducing equivalents $\left(\mathrm{H}_{2}\right)$ from reduced thioredoxin to a disulfide (S-S) group in an acceptor protein, which is converted to the protein-thiol (2SH) form, concomitantly with the oxidation of thioredoxin. In the dark, SH groups of reduced thioredoxin are oxidized to $\mathbf{S}-\mathrm{S}$, and oxidized thioredoxin oxidizes the reduced enzyme. Specific SH groups are involved in this redox mechanism in the different target enzymes. Key biosynthetic enzymes are activated on reduction and inactivated on oxidation, while key enzymes of carbohydrate degradation show an opposite response (deactivation on reduction and activation on oxidation). The system minimizes 'futile cycling' in chloroplasts, so that carbon dioxide assimilation occurs during the day and carbohydrate degradation takes place mostly at night [1].

A different possible level of redox modulation involving protein sulfhydryls in plants cells has been unveiled through the study of the expression of bacterial genes encoding cysteine-rich proteins in plant protoplasts $[2,7]$. The bacterial enzyme $\beta$-glucuronidase (GUS, 9 cysteines) was inactivated by oxidized thionins, cysteine-rich plant polypeptides, both in vitro and when thionins were externally added to tobacco protoplasts in which the enzyme was transgenically expressed [2]. Inactivation seemed to occur more rapidly in vivo, which suggested the mediation of some cellular redox mechanism [2]. This led to the observation that the expression of genes encoding cysteinerich bacterial proteins, such as GUS or neomycin phosphotransferase II (NPTII, 5 cysteines) could be increased up to 8-fold in response to externally added dithiothreitol (DTT) at concentrations that did not affect protoplast viability (up to $2.5 \mathrm{mM}$ ) [7]. Both the enzyme activity and the amount of enzyme were concomitantly increased by the treatment, whereas steady-state mRNA levels were not affected. DTT did not affect the activity of these enzymes in vitro (or in vivo, if it was already functional) but could overcome the inactivation by thionins, both in vitro and in protoplasts [7]. Expression of the gene for a cysteine-poor enzyme (i.e. phosphinothricin acetyl-transferase, 1 cysteine) was not affected. Similar increases in GUS and NPTII activities in response to DTT were also observed when these proteins were expressed with a signal peptide, which resulted in translocation to the endo- 
plasmic reticulum and secretion, although the overall range of expression levels were significantly below those obtained in the cytoplasm. Export was not affected by DTT. These observations led to the conclusion that redox changes could affect either a rate-limiting step in the folding of the nascent cysteine-rich proteins, which would determine their overall rate of synthesis, or that the more oxidizing environment would increase the proportion of misfolded proteins, which would be eventually degraded [7].

The translation machinery itself does not seem susceptible of redox modulation involving protein thiols, but recent findings indicate that the stability of mRNA can be affected by the redox state of proteins [8]. The mRNA encoding a bean proline-rich protein was shown to be destabilized in elicitor-treated cells, and this destabilization occurred simultaneously with the increase of a $50 \mathrm{kDa}$ protein that was specifically able to bind a 27-nucleotide U-rich sequence in the $3^{\prime}$ untranslated region of the messenger. Treatment with DTT or 2-mercaptoethanol increased binding activity, whereas oxidation or alkylation of the protein inhibited binding [8].

Further down the gene expression pathway, at the level of transcriptional or early postranscriptional events, there is also indirect evidence that redox changes in proteins play a regulatory role. It has been recently shown that the gene for $\mathrm{Cu} / \mathrm{Zn}$ superoxide dismutase is induced by DTT, glutathione, or cysteine [4], and that glutathione can mimic a fungal elicitor in the induction of the transient expression of the gene for chalcone synthase, a component of plant defense mechanism against microbial attack [3]. Many non-plant transcription factors (Fos, Jun, Ets, Rel, Myb, and others) have been found to lose their binding capacity on oxidation and to regain it by DTT treatment [see 6].

The above examples raise many specific questions for future research, but one that cuts across all of them is the identification and quantitation of the principal redox buffer in the different compartments of the plant cell, under normal growth (light/dark) and under various stress conditions, including oxidative stress. Although the ratio of reduced to oxidized glutathione (GSH/GSSG) is thought to play a key role in non-plant systems [see 5], the situation in plants is far from clear, especially in its quantitative aspects, as most measurements have dealt with total free thiols or with reduced glutathione. It has been repeatedly shown that formation and rearrangement of disulfide bonds are reversible S-S/SH exchange reactions that are kinetically and thermodynamically affected by the redox state of the medjum. In mammalian cells, the redox potential in the endoplasmic reticulum, GSH/GSSG ratio of $1: 1$ to $3: 1$, has been found to be much more oxidizing than in the cytosol, GSH/GSSG ratio of 30:1 to 100:1 [5]. Lack of this type of data for chloroplasts, endoplasmic reticulum, cytosol, or nuclear compartment of plant cells is limiting our present capacity to interpret redox regulatory phenomena in plants.

Some specific aspects related to the different levels of possible redox modulation that merit further investigation are, for example, the following: (1) possible regulation of genes encoding target enzymes of the thioredoxin system by translational and transcriptional mechanisms exemplified above; (2) effects of redox potentials on folding, assembly, and sorting of cysteine-rich plant proteins, under normal growth and under stress. This type of studies has been actively pursued in animal cells [refs. cited in 5, 7]; (3) occurrence in vivo, and generality of a proteinmediated redox modulation of mRNA stability; (4) redox modulation of plant transcriptional factors.

\section{References}

1. Buchanan $\mathrm{BB}$ : Regulation of $\mathrm{CO}_{2}$ assimilation in oxygenic photosynthesis: the ferredoxin/thioredoxin system. Arch Biochem Biophys 288: 1-9 (1991).

2. Diaz I, Carmona MJ, Garcia-Olmedo F: Effects of thionins on $\beta$-glucutonidase in vitro and in plant protoplasts. FEBS Lett 296: 279-282 (1992).

3. Dron M, Clouse SD, Dixon RA, Lawton MA, Lamb CJ: Glutathione and fungal elicitor regulation of a plant defense gene promoter in electroporated protoplasts. Proc Natl Acad Sci USA 85: 6738-6742 (1988).

4. Hérouart D, Van Montagu M, Inzé D: Redox-activated expression of the cytosolic copper/zinc superoxide dismu- 
tase gene in Nicotiana. Proc Natl Acad Sci USA 90: 31083112 (1993).

5. Hwang C, Sinskey AJ, Lodish HF: Oxidized redox state of glutathione in the endoplasmic reticulum. Science 257 : 1496-1501 (1992).

6. Myrset AH, Bostad A, Jamin N, Lirsac P-N, Toma F, Gabrielsen OS: DNA and redox state induced conformational changes in the DNA-binding domain of the $\mathrm{Myb}$ oncoprotein. EMBO J 12: 4625-4633 (1993).
7. Piñeiro M, Garcia-Olmedo F, Diaz I: Redox modulation of the expression of bacterial genes encoding cysteine-rich proteins in plant protoplasts. Proc Natl Acad Sci USA, 91: 3867-3871 (1994).

8. Zhang S, Medhy MC: Binding of a $50-\mathrm{kD}$ protein to a U-rich sequence in an mRNA encoding a proline-rich protein that is destabilized by fungal elicitor. Plant Cell 6: 135-145 (1994). 